\title{
Depth of valve implantation, conduction disturbances and pacemaker implantation with CoreValve and CoreValve Accutrak system for Transcatheter Aortic Valve Implantation, a multi-center study ${ }^{\text {is }}$
}

\author{
Guy D. Lenders ${ }^{\mathrm{a}, *, 1}$, Valérie Collas ${ }^{\mathrm{a}, 1}$, José Maria Hernandez ${ }^{\mathrm{b}, 1}$, Victor Legrand ${ }^{\mathrm{c}, 1}$, Haim D. Danenberg ${ }^{\mathrm{d}, 1}$, \\ Peter den Heijer ${ }^{\mathrm{e}, 1}$, Inez E. Rodrigus ${ }^{\mathrm{a}, 1}$, Bernard P. Paelinck ${ }^{\mathrm{a}, 1}$, Christiaan J. Vrints ${ }^{\mathrm{a}, 1}$, Johan M. Bosmans ${ }^{\mathrm{a}, 1}$ \\ a University Hospital Antwerp, Edegem, Belgium \\ ${ }^{\mathrm{b}}$ Hospital Universitario Virgen de la Victoria, Malaga, Spain \\ c $\mathrm{CHU}$ de Liege, Liege, Belgium

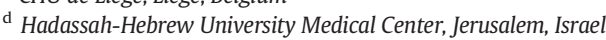 \\ e Amphia Hospital, Breda, The Netherlands
}

\section{A R T I C L E I N F O}

\section{Article history:}

Received 21 November 2013

Received in revised form 7 May 2014

Accepted 26 July 2014

Available online $\mathrm{xxxx}$

\section{Keywords:}

Transcatheter Aortic Valve Implantation (TAVI)

Left Bundle Branch Block (LBBB)

Pacemaker

Depth of valve implantation

\begin{abstract}
A B S T R A C T
Background: Transcatheter Aortic Valve Implantation (TAVI) is now considered an indispensable treatment strategy in high operative risk patients with severe, symptomatic aortic stenosis. However, conduction disturbances and the need for Permanent Pacemaker (PPM) implantation after TAVI with the CoreValve prosthesis still remain frequent.

Methods and results: We aimed to evaluate the implantation depth, the incidence and predictors of new conduction disturbances, and the need for PPM implantation within the first month after TAVI, using the new Accutrak CoreValve delivery system (ACV), compared to the previous generation CoreValve (non-ACV). In 5 experienced TAVI-centers, a total of 120 consecutive non-ACV and 112 consecutive ACV patients were included $(n=232)$. The mean depth of valve implantation (DVI) was $8.4 \pm 4.0 \mathrm{~mm}$ in the non-ACV group and $7.1 \pm 4.0 \mathrm{~mm}$ in the ACV group $(p=0.034)$. The combined incidence of new PPM implantation and new LBBB was $71.2 \%$ in the non-ACV group compared to $50.5 \%$ in the ACV group $(p=0.014)$. DVI $(p=0.002)$, first degree AV block $(\mathrm{p}=0.018)$ and RBBB $(\mathrm{p}<0.001)$ were independent predictors of PPM implantation. DVI $(\mathrm{p}<0.001)$ and pre-existing first degree AV-block $(p=0.021$ ) were identified as significant predictors of new LBBB.

Conclusion: DVI is an independent predictor of TAVI-related conduction disturbances and can be reduced by using the newer CoreValve Accutrak delivery system, resulting in a significantly lower incidence of new LBBB and new PPM implantation.
\end{abstract}

(c) 2014 Published by Elsevier Ireland Ltd.

\section{Introduction}

Transcatheter Aortic Valve Implantation (TAVI) has become the treatment of choice for inoperable patients with severe, symptomatic aortic stenosis and a valuable alternative for those with high operative risk [1-3]. However, TAVI performed with the self-expanding Medtronic CoreValve ${ }^{\circledR}$ device (CV) is frequently complicated by new conduction disturbances and subsequent need for Permanent Pacemaker (PPM) implantation. Due to intrinsic properties of the delivery catheter, the use of the first generation $18 \mathrm{Fr} \mathrm{CV}$ often resulted

\footnotetext{
Conflict of interest: José Maria Hernandez, Haim Danenberg, Victor Legrand, Peter den Heijer and Johan Bosmans are part-time clinical proctors for Medtronic CoreValve.

* Corresponding author at: University Hospital Antwerp, Wilrijkstraat 10, B-2650 Edegem, Belgium. Tel.: + 32382135 38; fax: + 3238214909.

E-mail address: guy.lenders@gmail.com (G.D. Lenders).

1 This author takes responsibility for all aspects of the reliability and freedom from bias of the data presented and their discussed interpretation.
}

in anatomically deep valve implantation. It has been hypothesized that trauma to the interventricular septum by deep insertion of the nitinol frame could impede the conduction tissue which it embeds, resulting in a high incidence of new Left Bundle Branch Block (LBBB) or complete AV-block after valve implantation [4,5]. This might explain the relatively high rate of new pacemaker implantation, as consistently reported by several TAVI registries [6,7].

The Accutrak delivery catheter for the CV has an additional stability layer by which friction forces are reduced, resulting in a more controlled and accurate positioning of the CV. Therefore it was hypothesized that using the Accutrak delivery catheter could result in a higher valve implantation, inflicting a milder trauma to surrounding structures and conduction tissue resulting in a decreased need of new pacemaker implantation. However, only few comparative data are available at present [8].

The pre-specified primary aim of this multi-center Accutrak conduction study was to evaluate the implantation depth, the incidence of new 
conduction disturbances, and the need for and indications of PPM implantation within the first month after TAVI, using the new Accutrak $\mathrm{CV}$ delivery system (ACV), compared to the previous generation $\mathrm{CV}$ (non-ACV) in a consecutive "real world" patient population. In addition, we tried to identify predictors for new PPM implantation and new LBBB occurrence.

\section{Methods}

\subsection{Study population}

A total of 232 patients were included in 5 high-volume centers experienced in performing TAVI with the CV prosthesis between January 2008 and May 2011. All centers were asked to include the first 20 to 25 consecutive patients before and after implementation of the Accutrak delivery system after excluding 5 "learning curve" patients. Finally, each center included 17 to 25 consecutive non-Accutrak patients (non-ACV group) and afterwards 21 to 25 consecutive Accutrak patients (ACV group). All patients had severe symptomatic aortic valve stenosis, with (very) high operative risk. TAVI implantation was considered feasible by the local heart team, consisting of cardiologists, interventional cardiologists, cardiac surgeons and anaesthesiologists. All patients underwent TAVI with the $\mathrm{CV}$ prosthesis.

\subsection{Data collection and processing}

Age, gender, logistic EuroSCORE, the mean gradient of the aortic stenosis, Aortic Valve Area (AVA), presence of atrial fibrillation (AF), pre-existing LBBB and RBBB, pre-existing 1st degree AV-block and previous PM implantation were assessed in all patients. ECG was performed before, after, at hospital discharge and at 1 month follow-up after TAVI. Vascular access site and size of the prosthesis were assessed for all procedures. Depth of valve implantation (DVI) was assessed in a central core lab (Antwerp University Hospital) based on procedural final angiography performed immediately after TAVI. Blinded measurements were made by one operator (VC), to ensure consistency of the results, using IMPAX Cardiovascular Information System (Agfa Healthcare, Mortsel, Belgium) and CAAS system (Pie Medical Imaging, Maastricht, The Netherlands) software. First, an angiographic projection in which the three sinuses of the aortic valve were seen in the same plane (annular perpendicular view) was chosen as a reference. Secondly, the lower edges of the three cusps of the aortic valve were connected to form a reference line. Perpendicular to this line, the implantation depth of the CoreValve prosthesis was measured medial (interventricular septum) and lateral. The mean of these two measurements was considered the correct DVI in the left ventricular outflow tract. DVI was quantitatively measured in mm beneath the aortic annulus and a mean depth of 4-6 mm beneath the aortic annulus was considered "normal". Fig. 1 shows a high (A) and low (B) TAVI implantation. Calibration was done using the 5 French pigtail-catheter as a reference. Intraclass correlation was used to test for reproducibility. A total of 15 random measurements were repeated with an intraclass correlation coefficient of 0.96 . One center (Malaga) performed, in a similar way, their own measurements of DVI both in ACV patients and nonACV patients, excluding possible bias.

New PPM-implantation was assessed until 30 days after the procedure and indications were classified according to the ESC-guidelines for PPM implantation [9].

\subsection{Endpoints}

The combined primary endpoint for the need for new PPM implantation and the occurrence of new LBBB (not requiring new PPM implantation), in the first month after TAVI, was compared between the ACV group and the non-ACV group. Secondly, DVI was compared between both groups and the relationship with significant conduction disturbances was assessed. Finally, possible predictors for the need of new PPM implantation and the occurrence of LBBB were analyzed.

\subsection{Statistical analysis}

Statistical analysis for the study was performed using SPSS software version 20.0 (IBM Corporation, New York). Categorical variables were expressed as percentages and were compared using Chi Square tests. Fisher's exact test was performed when applicable. Continuous variables were expressed as mean \pm SD and were compared using unpaired Student t-tests when normal distribution occurred. Mann-Whitney U-tests were used in case of abnormal distribution. Correlation between baseline characteristics and the CoreValve type were determined by binary logistic regression. All p-values were 2 -sided and differences were considered statistically significant when $\mathrm{p}<0.05$.

\section{Results}

\subsection{Baseline patient and procedural characteristics}

A total of 232 consecutive patients - 120 non-ACV and 112 ACV patients - were included between May 2007 and December 2011. Baseline patient and procedural characteristics are presented in Table 1. No significant differences in baseline characteristics between the two patient groups could be detected. The mean age was $81.7 \pm$ 6.0 years, mean logistic EuroSCORE was $22.2 \pm 14.3 \%$ and mean AVA was $0.62 \pm 0.16 \mathrm{~cm}$ [2], reflecting a typical TAVI population with severe aortic valve stenosis, old age and high risk for surgical valve replacement. Pre-procedural ECG-analysis demonstrated atrial fibrillation (AF) in 37 patients (15.9\%). Eighteen patients had already a PPM (7.8\%) and were excluded from analysis. RBBB was present in 26 patients $(12.10 \%)$, LBBB in another 26 patients $(12.1 \%)$ while 1 st degree AV block was present in 33 patients (15.4\%).

In the majority of patients TAVI was performed by femoral access (>90\%). Subclavian access was used in only 12 patients $(10 \%)$ in the non-ACV group and 8 patients (7\%) in the ACV group. Three patients in the ACV group were treated by direct aortic access. Both $26 \mathrm{~mm}$ valve size and $29 \mathrm{~mm}$ valve size were used almost equally in both the non-ACV and ACV groups. The $23 \mathrm{~mm}$ and $31 \mathrm{~mm} \mathrm{CV}$ devices were not yet widely available at the time of this study. Post dilatation was
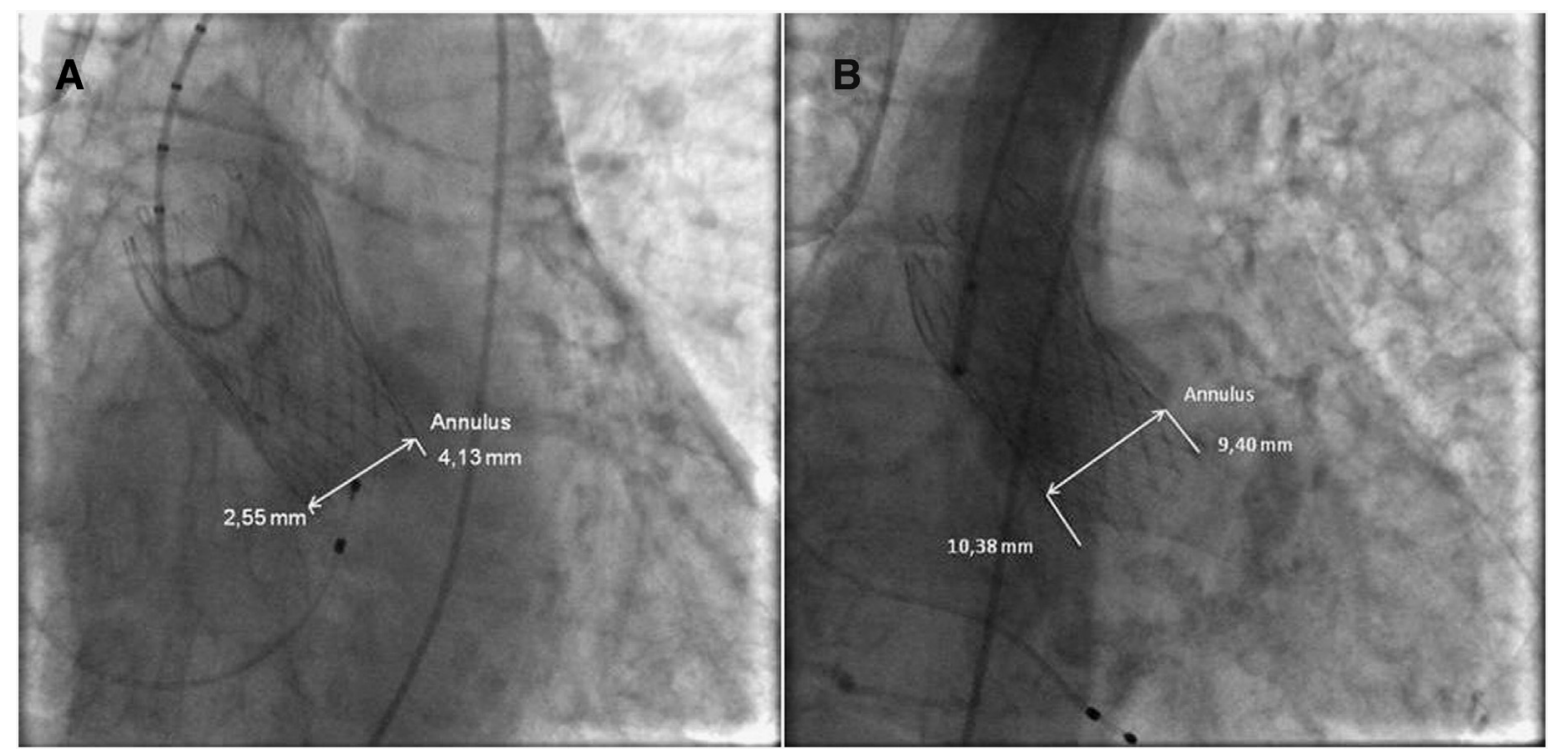

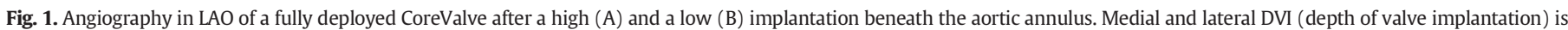
depicted. 
Table 1

Baseline and procedural characteristics. Categorical and continuous variables.

\begin{tabular}{|c|c|c|c|c|}
\hline & Non-AMCV $(\mathrm{n}=120)$ & $\operatorname{AMCV}(\mathrm{n}=112)$ & Total $(\mathrm{n}=232)$ & p-Value \\
\hline Age (years) - mean $\pm S D$ & $82.0 \pm 5.2$ & $81.4 \pm 6.8$ & $81.7 \pm 6.0$ & 0.412 \\
\hline Male - n (\%) & $56(46.7)$ & $45(40.2)$ & $101(43.5)$ & 0.319 \\
\hline Logistic EuroSCORE $(\%)-$ mean \pm SD & $21.1 \pm 12.6$ & $23.5 \pm 15.9$ & $22.2 \pm 14.3$ & 0.466 \\
\hline $\operatorname{AVA}\left(\mathrm{cm}^{2}\right)-$ mean $\pm \mathrm{SD}$ & $0.64 \pm 0.14$ & $0.61 \pm 0.18$ & $0.62 \pm 0.16$ & 0.157 \\
\hline Mean gradient $\mathrm{AV}(\mathrm{mm} \mathrm{Hg})-$ mean $\pm \mathrm{SD}$ & $47.7 \pm 14.3$ & $47.7 \pm 13.1$ & $47.7 \pm 13.7$ & 0.968 \\
\hline Previous $\mathrm{AF}-\mathrm{n}(\%)$ & $16(13.3)$ & $21(18.8)$ & $37(15.9)$ & 0.245 \\
\hline Previous pacemaker $-\mathrm{n}(\%)$ & $10(8.3)$ & $8(7.14)$ & $18(7.8)$ & 0.735 \\
\hline $\mathrm{RBBB}-\mathrm{n}(\%)$ & $14(11.7)$ & $12(10.7)$ & $26(11.2)$ & 0.832 \\
\hline LBBB $-\mathrm{n}(\%)$ & $15(12.5)$ & $11(9.8)$ & $26(11.2)$ & 0.528 \\
\hline 1st degree AV block - n (\%) & $18(15)$ & $15(13.4)$ & $33(14.2)$ & 0.781 \\
\hline Transfemoral - n (\%) & $108(90.0)$ & $101(90.2)$ & $209(90.1)$ & 0.964 \\
\hline Transsubclavian $-\mathrm{n}(\%)$ & $12(10.0)$ & $8(7.1)$ & $20(8.6)$ & 0.438 \\
\hline Direct aortic - n (\%) & $0(0)$ & $3(2.7)$ & $3(1.3)$ & 0.111 \\
\hline 26 mm CoreValve - n (\%) & $49(40.8)$ & $54(48.2)$ & $103(44.4)$ & 0.258 \\
\hline $29 \mathrm{~mm}$ CoreValve - n (\%) & $71(59.2)$ & $58(51.8)$ & $129(55.6)$ & 0.205 \\
\hline Post dilatation $-\mathrm{n}(\%)$ & $20(16.7)$ & $24(21.4)$ & $44(18.9)$ & 0.324 \\
\hline
\end{tabular}

AMCV: Accutrack Medtronic CoreValve; AVA: Aortic valve area; AF: Atrial fibrillation; RBBB: Right Bundle Branch Block; LBBB: Left Bundle Branch Block.

performed equally in both groups $(16.7 \%$ in the non-ACV group and $21.4 \%$ in the ACV group).

\subsection{Depth of valve implantation, conduction disturbances and pacemaker implantation}

Table 2 summarizes the mean DVI and the incidence of new PPM implantations, new conduction disturbances and mortality at 30 days. The mean DVI was $8.4 \pm 4.0 \mathrm{~mm}$ in the non-ACV group and $7.1 \pm 4.0 \mathrm{~mm}$ in the ACV group $(\mathrm{p}=0.034)$. In patients without PPM or LBBB before valve implantation, the combined incidence of new PPM implantation and new LBBB (primary endpoint) was $71.2 \%$ in the non-ACV group compared to $50.5 \%$ in the ACV group $(\mathrm{p}=0.014)$. In the non-ACV group, 33 (32.3\%) "pacemaker naïve" patients received PPM after valve implantation compared to 21 (21.4\%) "pacemaker naïve" patients in the ACV group $(\mathrm{p}=0.094)$. In the non-ACV group, 29 (33.3\%) "LBBB-naïve patients" developed new LBBB without a need for new PPM at discharge, compared to 23 (26.4\%) "LBBB-naïve patients" in the ACV group ( $p=0.320)$. The occurrence of a new RBBB in "RBBB-naïve patients" was rare and did not differ between both groups $(p=1.000)$ nor did the occurrence of new 1 st degree AV-block after valve implantation $(p=0.909)$. The 30 day all-cause mortality was low and not significantly different between the non-ACV group (6.7\%) and the ACV group (5.4\%).

Most pacemakers were implanted because of 3rd degree AV-block (67\% in the non-ACV group and 76\% in the ACV group) without any statistical difference in all classes of indications for pacemaker implantation (Table 3). Most PPMs were implanted in the first week after TAVI, both in the ACV and non-ACV groups.

Table 2

DVI, new Permanent Pacemaker (PPM) implantations, conduction disturbances and allcause mortality, 30 days after TAVI.

\begin{tabular}{|c|c|c|c|c|}
\hline & Non-AMCV & AMCV & Total & p-Value \\
\hline $\mathrm{DVI}(\mathrm{mm})-$ mean $\pm \mathrm{SD}$ & $8.4 \pm 4.0$ & $7.1 \pm 4.0$ & $7.7 \pm 4.1$ & 0.034 \\
\hline New PPM + new LBBB $-\mathrm{n}(\%)^{a}$ & $73(76.8)$ & $53(56.9)$ & $126(67.0)$ & 0.014 \\
\hline New PPM - n (\%) ${ }^{b}$ & $32(29.1)$ & $21(20.2)$ & $53(24.8)$ & 0.082 \\
\hline New LBBB $-\mathrm{n}(\%)^{\mathrm{a}}$ & $41(43.2)$ & $32(34.4)$ & $73(38.8)$ & 0.053 \\
\hline New $R B B B-n(\%)^{c}$ & $1(1.0)$ & $4(4.3)$ & $5(2.7)$ & 0.368 \\
\hline New 1st degree AV block $-\mathrm{n}(\%)^{\mathrm{d}}$ & $13(17.1)$ & $15(22.1)$ & $28(19.4)$ & 0.742 \\
\hline All-cause 30 day mortality - n (\%) & $8(6.7)$ & $6(5.4)$ & $14(6.0)$ & 0.676 \\
\hline
\end{tabular}

AMCV: Accutrack Medtronic CoreValve; PPM: Permanent Pacemaker; LBBB: Left Bundle Branch Block; RBBB: Right Bundle Branch Block; AV: Atrio Ventricular. Bold values indicate significance at $\mathrm{p}<0.05$.

a Patients with previous PPM or previous LBBB excluded $(\mathrm{n}=188$ ).

b Patients with previous PPM excluded $(\mathrm{n}=214)$.

c Patients with previous PPM or previous RBBB excluded $(\mathrm{n}=188)$.

d Patients with previous PPM. 1st degree AV-block and AF excluded $(\mathrm{n}=144)$.
The relationship between DVI and the need for new PPM implantation, for both the non-ACV and ACV groups, is presented in Table 4 and Fig. 2. The mean DVI in all patients (combined ACV and nonACV) that required new PPM implantation $(n=54)$ was $8.9 \pm$ $4.2 \mathrm{~mm}$, which is significantly lower compared to those who did not need new PPM implantation with a mean DVI of $6.9 \pm 3.8 \mathrm{~mm}$ $(p=0.002)$. In the non-ACV group this difference remains significant with a mean DVI of $9.4 \pm 4.2 \mathrm{~mm}$ in patients receiving PPM implantation compared to $7.3 \mathrm{~mm} \pm 3.7$ in those who did not $(\mathrm{p}=$ 0.022 ). A trend remained also in the ACV group, with the mean DVI of $8.2 \pm 4.2 \mathrm{~mm}$ in patients receiving pacemaker implantation compared to $6.6 \mathrm{~mm} \pm 4.1$ in those who did not $(\mathrm{p}=0.115)$.

The relationship between DVI and new LBBB is also statistically significant with a lower implantation in the patients with a new LBBB $(8.8 \pm 3.5 \mathrm{~mm})$ compared with those without a new LBBB $(5.4 \pm 3.5 \mathrm{~mm})$. This difference remains statistically significant in both the non-ACV group and the ACV group (Table 5).

\subsection{Predictors}

In a univariate analysis of the total population of both ACV and non$A C V$ patients, pre-existing 1 st degree AV block ( $p=0.018$ ), preexisting RBBB $(\mathrm{p}<0.001)$ and DVI $(\mathrm{p}=0.002)$ were predictors of new PPM implantation at 30 days after TAVI (Table 6). In Table 7, a similar univariate analysis of the total study population is depicted for the occurrence of a new LBBB (only significant predictors and the use of the Accutrak catheter are shown). DVI and pre-existing 1st degree AV-block were identified as significant predictors.

\section{Discussion}

The pre-specified primary aim of this multi-center conduction study was to evaluate the implantation depth, the incidence of new conduction disturbances, and the need for and indications of PPM implantation

Table 3

Indications for new PPM implantation after TAVI.

\begin{tabular}{lcccc}
\hline & $\begin{array}{l}\text { Non-ACV } \\
(\mathrm{n}=32)\end{array}$ & $\begin{array}{l}\text { ACV } \\
(\mathrm{n}=21)\end{array}$ & $\begin{array}{l}\text { Total } \\
(\mathrm{n}=53)\end{array}$ & p-Value \\
\hline 3rd degree AV block - $\mathrm{n}(\%)$ & $21(65.6)$ & $16(76.2)$ & $37(69.8)$ & 0.412 \\
New LBBB $-\mathrm{n}(\%)$ & $3(9.4)$ & $3(14.3)$ & $6(11.3)$ & 1.000 \\
New 1st degree AV block - $\mathrm{n}(\%)$ & $1(3.1)$ & $0(0)$ & $1(1.9)$ & 0.625 \\
1st degree AV block + LBBB - $\mathrm{n}(\%)$ & $4(12.5)$ & $0(0)$ & $4(7.5)$ & 0.143 \\
Significant bradycardia - $\mathrm{n}(\%)$ & $3(9.4)$ & $2(9.5)$ & $5(9.4)$ & 0.755 \\
\hline
\end{tabular}

PPM: Permanent Pacemaker; ACV: Accutrack CoreValve; AV: Atrio Ventricular; LBBB: Left Bundle Branch Block. 
Table 4

Depth of valve implantation (DVI) versus PPM.

\begin{tabular}{llll}
\hline & $\begin{array}{l}\text { DVI }- \text { no PPM } \\
\text { Mean } \pm \text { SD }(\mathrm{mm})\end{array}$ & $\begin{array}{l}\text { DVI }- \text { PPM } \\
\text { Mean } \pm \text { SD }(\mathrm{mm})\end{array}$ & p-Value \\
\hline Non-ACV & $7.3 \pm 3.7$ & $9.4 \pm 4.2$ & $\mathbf{0 . 0 2 2}$ \\
ACV & $6.6 \pm 4.1$ & $8.2 \pm 4.2$ & 0.115 \\
Total & $\mathbf{6 . 9} \pm \mathbf{3 . 8}$ & $\mathbf{8 . 9} \pm \mathbf{4 . 2}$ & $\mathbf{0 . 0 0 2}$ \\
\hline
\end{tabular}

PPM: Permanent Pacemaker; ACV: Accutrack CoreValve.

Bold values indicate significance at $\mathrm{p}<0.05$.

within the first month after TAVI, using the new Accutrak CV delivery system, compared to the previous generation CV in a consecutive "real world" patient population. In addition, we aimed to identify predictors of the occurrence of new PPM implantation and new LBBB. The main findings of this study can be summarized as follows:

(1) The incidences of new PPM implantation and new LBBB are confirmed to be high after TAVI, especially when using the "old generation" non-Accutrak $18 \mathrm{Fr}$ CV prosthesis.

(2) The mean depth of valve implantation is confirmed to be a strong predictor, for both new PPM implantation and new LBBB after TAVI.

(3) The mean depth of valve implantation is significantly reduced using the "new generation" Accutrak 18Fr CV prosthesis.

(4) The implementation of the "new generation" Accutrak $18 \mathrm{Fr}$ CV prosthesis significantly reduced the incidence of the combined endpoint of new PPM implantation and new LBBB after TAVI.

New conduction disturbances after TAVI remain a major limitation, especially with the self-expanding $C V$, with a reported incidence of PPM implantation varying between 16 and 40\% [10-13]. PPM implantation holds certain procedural risks, especially in an old and frail population such as the one that undergoes TAVI (in casu pneumothorax, bleeding, infection, etc.). Moreover, longstanding RV-pacing on itself can induce heart failure [14], or prevent left ventricular recovery. Furthermore, the occurrence of conduction disturbances not requiring PPM implantation after TAVI is high as well. In particular, LBBB has recently been reported to be associated with worse clinical outcome [15]. Trauma to the IV-septum and subsequently to the conduction tissue it embeds, induced by the relatively deep implanted valve frame, is considered to be a major determinant for the new conduction disturbances occurring after TAVI [16]. Awareness of this post procedural

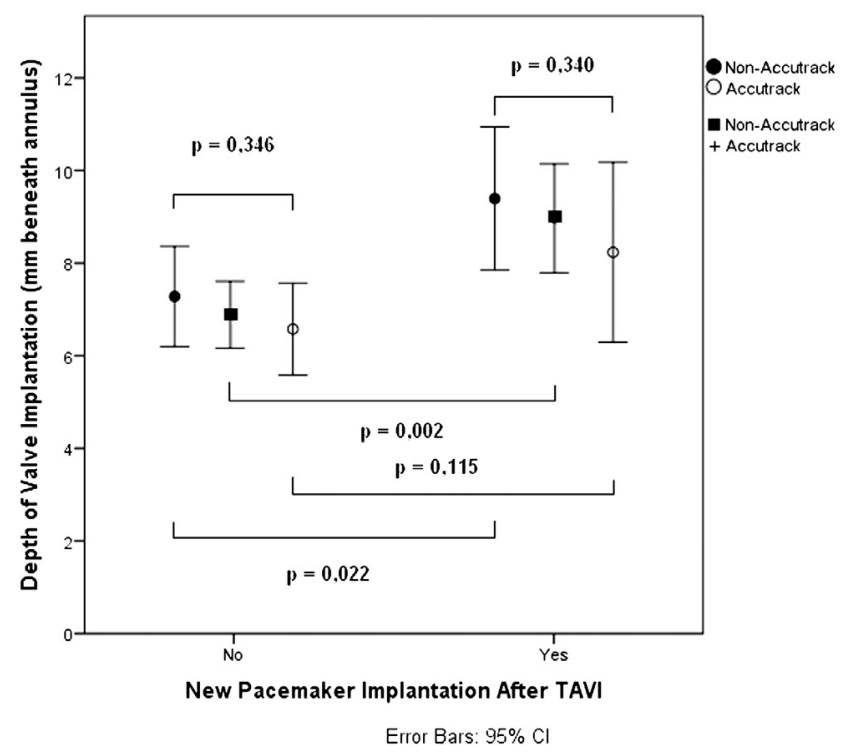

Fig. 2. Depth of valve implantation in patients with and without the need for new Pacemaker implantation. Clustered error bars of Accutrack patients, non-Accutrack patients and the combination of both Accutrack and non-Accutrack patients are shown.
Table 5

Depth of valve implantation (DVI) versus new LBBB.

\begin{tabular}{lllr}
\hline & $\begin{array}{l}\text { DVI }- \text { no LBBB } \\
\text { mean } \pm \text { SD }(\mathrm{mm})\end{array}$ & $\begin{array}{l}\text { DVI }- \text { LBBB } \\
\text { mean } \pm \text { SD }(\mathrm{mm})\end{array}$ & p-Value \\
\hline Non-ACV & $6.15 \pm 2.97$ & $8.79 \pm 4.09$ & $\mathbf{0 . 0 2 2}$ \\
ACV & $4.97 \pm 3.78$ & $8.82 \pm 2.92$ & $<\mathbf{0 . 0 0 1}$ \\
Total & $\mathbf{5 . 4 0} \pm \mathbf{3 . 5 2}$ & $\mathbf{8 . 8 1} \pm \mathbf{3 . 5 1}$ & $<\mathbf{0 . 0 0 1}$ \\
\hline
\end{tabular}

LBBB: Left Bundle Branch Block; ACV: Accutrack CoreValve.

Bold values indicate significance at $\mathrm{p}<0.05$.

complication and its underlying pathophysiology has resulted in caution and attempts by operators to perform a safer and thus higher valve implantation. However proper valve positioning has been shown to be relatively challenging using the "old generation" CoreValve.

Several predictors for PPM implantation after TAVI have been identified, mostly in studies restricted by a relatively small sample size. Pre-existing RBBB, depth of valve implantation, hypertrophy of the interventricular septum and pre-existing 1st degree AVblock have previously been identified as predictors for the need for new PPM implantation after TAVI $[5,17,18]$. In this relatively large, multi-center study, not only depth of valve implantation, but also pre-existing RBBB and pre-existing 1st degree AV-block confirmed to be strong predictors of the need for new PPM implantation. Better control of depth of valve implantation, especially for the $\mathrm{CV}$, is therefore of great clinical relevance in the prevention of conduction disturbances after TAVI.

There is no clear methodological consensus on how to measure most accurately DVI below the annulus. In the single center study by MuñozGarcía et al. [19], the distance from the non-coronary cusp to the distal extreme of the prosthesis, based on angiography after TAVI, was considered to be the implantation depth. Using this methodology, the Accutrak delivery system was associated with less deep prosthesis implantation in the left ventricular outflow tract, which could be related to the lower rate of PPM requirement. Also Piazza et al. [20] demonstrated that the mean distance between the proximal end of the CV prosthesis and the lower edge of the non-coronary sinus was significantly shorter in patients who did not need PPM implantation. On the other hand, Tchetche et al. [8] defined DVI as the maximal distance between the intraventricular end of the prosthesis and the aortic annulus at the level of the non-coronary cusp and the left anterior coronary cusp. In this study, evaluating exclusively the Accutrak system, the mean DVI was $4.9 \pm 2 \mathrm{~mm}$. In our actual study, we defined DVI as the mean of the distance from the nadir of the non-coronary and left coronary sinus to the ventricular edge of the frame, demonstrating that DVI is an independent predictor of TAVI-related conduction disturbances and can be reduced by using the newer CoreValve Accutrak delivery system. However, although different methodologies are reported for quantifying depth of valve implantation, complicating interpretation of absolute implantation depths, a consistent relation between DVI and occurrence of new conduction disturbances is confirmed by most authors.

Table 6

Predictors of new PPM implantation - univariate analysis. Bold values indicate significance at $\mathrm{p}<0.05$.

\begin{tabular}{lllr}
\hline & No new PPM & New PPM & p-Value \\
\hline AVA $\left(\mathrm{cm}^{2}\right)-$ mean \pm SD & $0.61 \pm 0.16$ & $0.65 \pm 0.17$ & 0.122 \\
Male $-\mathrm{n}(\%)$ & $54(37)$ & $27(50)$ & 0.096 \\
Pre-existing AVB I $-\mathrm{n}(\%)$ & $17(14.4)$ & $13(31)$ & $\mathbf{0 . 0 1 8}$ \\
Pre-existing LBBB $-\mathrm{n}(\%)$ & $21(14.5)$ & $4(7.4)$ & 0.181 \\
Pre-existing RBBB $-\mathrm{n}(\%)$ & $5(3.4)$ & $19(35.2)$ & $<\mathbf{0 . 0 0 1}$ \\
DVI (mm beneath annulus) $-\mathrm{n}(\%)$ & $6.87 \pm 3.92$ & $8.94 \pm 4.19$ & $\mathbf{0 . 0 0 2}$ \\
Accutrack $-\mathrm{n}(\%)$ & $77(52.7)$ & $21(38.9)$ & 0.082 \\
\hline
\end{tabular}

PPM: Permanent Pacemaker; AVA: Aortic Valve Area; AVB: Atrio Ventricular Block; LBBB: Left Bundle Branch Block; RBBB: Right Bundle Branch Block; DVI: depth of valve implantation.

Bold values indicate significance at $\mathrm{p}<0.05$. 
Table 7

Predictors of new LBBB - univariate analysis.

\begin{tabular}{lllr}
\hline & No new LBBB & New LBBB & p-Value \\
\hline DVI (mm beneath annulus) $-\mathrm{n}(\%)$ & $5.4 \pm 3.52$ & $8.81 \pm 3.51$ & $<\mathbf{0 . 0 0 1}$ \\
Pre-existing AVB I - n (\%) & $3(5.4)$ & $9(20.5)$ & $\mathbf{0 . 0 2 1}$ \\
Accutrack - n (\%) & $42(60)$ & $23(44.2)$ & 0.084 \\
\hline
\end{tabular}

LBBB: Left Bundle Branch Block; DVI: depth of valve implantation; AVB: Atrio Ventricular Block.

Bold values indicate significance at $\mathrm{p}<0.05$.

To the best of our knowledge, the actual Accutrak study is the first multicenter comparative study, performed in 5 high-volume TAVI centers, in which depth of valve implantation, both of the "old" and "new generation" $18 \mathrm{Fr} \mathrm{CV}$, is measured by a blinded core lab and related to the occurrence of new conduction abnormalities and new PPM implantation.

\subsection{Study limitations}

The present study is of observational nature with the inherent limitations of such a design. However, all patients in all centers have been included consecutively, without exclusion, representing a "real world" situation. In addition, the fact that all patients in the Accutrak group were treated later in time, compared to those treated with the older system, could have created a bias because the concept of DVI, being potentially responsible for conduction disturbances, may have resulted in attempts of operators to implant higher and thus safer in later procedures, independent of the new delivery device. We tried to minimize this potential bias by including the last patients treated with the previous delivery system and the first patients treated with the Accutrak delivery system in every center, reducing the difference in time to a minimum.

We also did not implement data about the valve/annulus ratio, an additional potential risk factor for post procedural conduction disturbances because in different centers, different imaging techniques were used for valve sizing.

A higher valve implantation could hypothetically result in more severe aortic regurgitation although we found no significant difference in our study. But since assessment of aortic regurgitation, in our study, was done with visual assessment at final angiography only, we did not implement these data in our study.

Also the use of a combined endpoint of new PPM implantation and new LBBB (without PPM implantation) can appear heterogeneous but both reflect very similar CV related conduction abnormalities and are important predictors of morbidity and even mortality, so can be taken in account together.

\section{Conclusion}

The incidences of TAVI-related new LBBB and new PPM implantation remain high with the Medtronic CoreValve system. DVI is a significant predictor for PPM implantation and new LBBB. The newer Accutrak delivery catheter can significantly reduce DVI, resulting in a significantly lower incidence of new LBBB and new PPM implantation. Further equivocal improvements in the conceptual design are needed to reduce complications if further indications for TAVI are to be extended.

\section{References}

[1] Leon MB, Smith CR, Mack M, et al. Transcatheter aortic-valve implantation for aortic stenosis in patients who cannot undergo surgery. N Engl J Med 2010:363:1597-607.

[2] Smith CR, Leon MB, Mack MJ, et al. Transcatheter versus surgical aortic-valve replacement in high-risk patients. N Engl J Med 2011;364:2187-98.

[3] Vahanian A, Alfieri O, Andreotti F, et al. Guidelines on the management of valvular heart disease (version 2012). Eur Heart J 2012;33:2451-96.

[4] Baan Jr J, Yong ZY, Koch KT, et al. Factors associated with cardiac conduction disorders and permanent pacemaker implantation after percutaneous aortic valve implantation with the CoreValve prosthesis. Am Heart J 2010;159:497-503.

[5] Guetta V, Goldenberg G, Segev A, et al. Predictors and course of high-degree atrioventricular block after transcatheter aortic valve implantation using the CoreValve Revalving system. Am J Cardiol 2011;108:1600-5.

[6] Khawaja MZ, Rajani R, Cook A, et al. Permanent pacemaker insertion after CoreValve transcatheter aortic valve implantation: incidence and contributing factors (the UK CoreValve Collaborative). Circulation 2011;123:951-60.

[7] Rodes-Cabau J, Webb JG, Cheung A, et al. Transcatheter aortic valve implantation for the treatment of severe symptomatic aortic stenosis in patients at very high or prohibitive surgical risk: acute and late outcomes of the multicenter Canadian experience. J Am Coll Cardiol 2010;55:1080-90.

[8] Tchetche D, Modine T, Farah B, et al. Update on the need for a permanent pacemaker after transcatheter aortic valve implantation using the CoreValve ${ }^{\circledR}$ Accutrak $^{\mathrm{TM}}$ system. Eurolntervention 2012;8:556-62.

[9] Vardas P, Auricchio A, Blanc JJ, et al. Guidelines for cardiac pacing and cardiac resynchronization therapy: the task force for cardiac pacing and cardiac resynchronization therapy of the European Society of Cardiology. Developed in collaboration with the European Heart Rhythm Association. Eur Heart J 2007;28: 2256-95.

[10] Tamburino C, Capodanno D, Ramondo A, et al. Incidence and predictors of early and late mortality after transcatheter aortic valve implantation in 663 patients with severe aortic stenosis. Circulation 2011:123:299-308.

[11] Piazza N, Nuis R, Tzikas A, et al. Persistent conduction abnormalities and requirements for pacemaking six months after transcatheter aortic valve implantation. Eurolntervention 2010;6:475-84.

[12] Piazza N, Grube E, Gerckens U, et al. Procedural and 30-day outcomes following transcatheter aortic valve implantation using the third generation (18 Fr) CoreValve revalving system: results from the multicentre, expanded evaluation registry 1 -year following CE mark approval. EuroIntervention 2008;4:242-9.

[13] Erkapic D, De Rosa S, Kelava A, Lehmann R, Fichtlscherer S, Hohnloser SH. Risk for permanent pacemaker after transcatheter aortic valve implantation: a comprehensive analysis of the literature. J Cardiovasc Electrophysiol 2011;3: 1540-8167.

[14] Nielsen JC, Andersen HR, Thomsen PE, et al. Heart failure and echocardiographic changes during long-term follow-up of patients with sick sinus syndrome randomized to single-chamber atrial or ventricular pacing. Circulation 1998;97:987-95.

[15] Houthuizen P, Van Garsse LA, Poels TT, et al. Left bundle-branch block induced by transcatheter aortic valve implantation increases risk of death. Circulation 2012; 126:720-8.

[16] Nuis RJ, Van Mieghem NM, Schultz CJ, et al. Timing and potential mechanisms of new conduction abnormalities during the implantation of the Medtronic CoreValve system in patients with aortic stenosis. Eur Heart J 2011;32:2067-74.

[17] Fraccaro C, Buja G, Tarantini G, et al. Incidence, predictors, and outcome of conduction disorders after transcatheter self-expandable aortic valve implantation. Am J Cardiol 2011;107:747-54

[18] Jilaihawi H, Chin D, Vasa-Nicotera M, et al. Predictors for permanent pacemaker requirement after transcatheter aortic valve implantation with the CoreValve bioprosthesis. Am Heart J 2009;157:860-6.

[19] Muñoz-García A, Hernández-García J, Jiménez-Navarro M, et al. Factors predicting and having an impact on the need for a permanent pacemaker after CoreValve prosthesis implantation using the new Accutrak delivery catheter system. JACC Cardiovasc Interv 2012;5:533-9.

[20] Piazza N, Onuma Y, Jesserun E, et al. Early and persistent intraventricular conduction abnormalities and requirements for pacemaking after percutaneous replacement of the aortic valve. JACC Cardiovasc Interv 2008;1:310-6. 